\title{
Subcapsular Splenic Urinoma and Splenorenal Fistula: A New Complication of Percutaneous Nephrolithotomy
}

\author{
Eugene B. Cone, MD, Ghalib Jibara, MD, MPH, Daniel Wollin, MD, and Glenn M. Preminger, MD
}

\begin{abstract}
Background: Splenic urinoma has not been previously reported as a complication of percutaneous nephrolithotomy (PNL).

Case Presentation: A 46-year-old Caucasian male underwent PNL for two large left renal stones. Surgery was performed in prone split-leg position, with access obtained through combined fluoroscopic guidance and direct ureteroscopic observation of the targeted calix. The tract was dilated to $30 \mathrm{~F}$ using a pressure balloon, and complete clearance of stones was obtained through a combination of rigid and flexible nephroscopy, as well as antegrade ureteroscopy. The patient was left with a Double-J ureteral stent on a string for removal in 1 week. After overnight observation, his hemoglobin, white blood cell count, and renal function were normal and thus he was discharged home. The day after stent removal, he presented to the emergency department with abdominal pain, was found to have mild left hydronephrosis on CT, and was discharged on 1 week of antibiotics. One week later he re-presented with worsening abdominal pain and was found to have an elevated creatinine and a white blood cell count of $21 \times 10^{9} / \mathrm{L}$. On contrasted CT with delayed images, an $18 \mathrm{~cm}$ splenic fluid collection was seen with a fistulous connection to the left kidney collecting system. He underwent emergent stent placement and improved clinically without drainage of the urinoma. A renal ultrasonography 1 month after stent placement confirmed resolution of the splenic urinoma, so the stent was removed at 1 month. Followup CT 1 month after stent removal was normal without any evidence of fistula or urinoma recurrence.

Conclusion: This is the first report of a subcapsular splenic urinoma and splenorenal fistula post-PNL. Ureteral stent placement was sufficient for drainage and to resolve the complication.
\end{abstract}

Keywords: nephrolithiasis, urolithiasis, percutaneous nephrolitothomy, percutaneous nephrolithotomy complication, urinoma, kidney trauma

\section{Introduction and Background}

$\mathbf{P}$ ERCUTANEOUS NEPHROLITHOTOMY (PNL) IS a wellestablished and relatively safe treatment for large burden nephrolithiasis. Although some series have reported as much as an $83 \%$ complication rate, the vast majority are minor (Clavien I or II), most commonly extravasation (7.2\%), transient decrease in renal function $(1.4 \%-16.9 \%)$, and fever $(21.0 \%-32.1 \%)$. Most complications are able to be managed conservatively or with minimally invasive options (i.e., percutaneous or internal drainage and intervention by interventional radiology). ${ }^{1}$ Although review articles have previously reported splenic injuries associated with PNL, the injuries reported typically relate to direct damage to the spleen during percutaneous access, such as hemorrhage, hematoma, or other bleeding complications managed either conservatively or with splenectomy. ${ }^{1,2}$ We report the first splenic urinoma resulting from a splenorenal fistula after PNL.

\section{Case Presentation}

\section{Patient}

A 46-year-old Caucasian male with a history of recurrent calcium oxalate stone formation was referred to our institution for treatment of a $2.5 \mathrm{~cm}$ left renal pelvis stone as well as a $1.5 \mathrm{~cm}$ collection of stones in the left lower pole. His presenting symptoms included intermittent left flank pain, but he denied fevers, dysuria, or urinary tract infections. He

Division of Urology, Duke Department of Surgery, Duke University School of Medicine, Durham, North Carolina.

(C) Eugene B. Cone et al. 2017; Published by Mary Ann Liebert, Inc. This is an Open Access article distributed under the terms of the Creative Commons Attribution License, which permits unrestricted use, distribution, and reproduction in any medium, provided the original work is properly cited. 
underwent shock wave lithotripsy at an outside hospital that was unsuccessful at clearing either stone. He was on no medical stone management, and his only medical history was hypertension treated with angiotensin converting enzyme inhibitor. His preoperative physical examination, vitals, laboratory studies, and urine culture were within normal limits with the exception of a BMI of $33 \mathrm{~kg} / \mathrm{m}^{2}$. Noncontrasted prone CT of the abdomen/pelvis (CT stone protocol) revealed the aforementioned stones and no other relevant pathology report (Fig. 1). The stones measured 1100-1300 HU, and no hydronephrosis or splenomegaly was noted. After extensive discussion of risks and benefits, the patient elected to undergo PNL.

\section{Intervention}

The patient was positioned in prone split-leg position, and his left ureter was catheterized using a 5F open-ended catheter during flexible cystoscopy. Retrograde pyelography revealed normal upper tract anatomy and the two large stones. After placement of an $11 \mathrm{~F} / 13 \mathrm{~F}$ ureteral access sheath, an $8 \mathrm{~F}$ digital flexible ureteroscope was navigated past the left renal pelvis stone, and a middle-posterior calix was identified with adequate access to both the renal pelvis and the lower pole. A retrograde air pyelogram confirmed the posterior calix that was accessed under direct ureteroscopic observation, confirming optimal placement of the needle in the center of the caliceal papilla. The tract was dilated to $30 \mathrm{~F}$ using a NephroMax High Pressure Balloon Catheter (Boston Scientific), after which a $30 \mathrm{~F}$ Amplatz working sheath was placed. The stones were identified, fragmented, and removed completely using an ultrasonic lithotrite (Olympus LUS-II). Complete clearance of both stones was confirmed using fluoroscopy, flexible nephroscopy (18F), and antegrade ureteroscopy $(8 \mathrm{~F})$. The access sheath was removed under direct ureteroscopic observation with only Grade I mucosal trauma noted to the ureter, so an internal ureteral stent on a string was placed retrograde under fluoroscopic guidance. Operative time was roughly 90 minutes and blood loss was $<50 \mathrm{~mL}$. No nephrostomy tube was left postoperatively. The patient was observed in the hospital overnight and discharged on postoperative day 1 with hemoglobin/hematocrit $(\mathrm{H} / \mathrm{H})$, white blood cell count, and creatinine all within normal limits. The patient was instructed to remove his Double-J ureteral stent at home after 1 week.

\section{Complication}

The patient removed his stent on postoperative day 7 . The next day he presented to his local emergency department (ED) with abdominal pain. His blood work was within normal limits except for a white blood cell count of $12 \times 10^{9} / \mathrm{L}$ (H/H was 11/35 from 10/32 postoperatively). A noncontrasted CT scan revealed mild left hydronephrosis and no residual stones. He was sent home on a 1-week course of ciprofloxacin.

Six days later he presented to our ED with worsening flank pain with a creatinine of $2.0 \mathrm{mg} / \mathrm{dL}$ (baseline 1.1 ), a white blood cell count of $21 \times 10^{9} / \mathrm{L}$, and $\mathrm{H} / \mathrm{H}$ of $11 / 34$. After fluid resuscitation in the $\mathrm{ED}$, his creatinine dropped to $1.4 \mathrm{mg} / \mathrm{dL}$ and his white blood cell count to $17 \times 10^{9} / \mathrm{L}$, but since his abdominal pain persisted, a CT urogram was obtained. The CT showed a large $18 \times 16 \times 8 \mathrm{~cm}$ subcapsular splenic fluid collection with a clearly delineated, opacified fistulous connection to the left kidney on delayed images (Fig. 2). Given the density of that fluid collection on CT scan, the lack of clinical and radiologic signs of abscess, and the lack of clinical signs of bleeding or hematoma, this finding was believed to be a urinoma with fistulous communication to the left renal collecting system.

General surgery was consulted and discussion between all involved services led to the conclusion that percutaneous splenic drainage was not urgently indicated. In addition, there was concern that placement of a percutaneous splenic drain carried a high risk of splenic injury. An emergent internal ureteral stent was placed to divert urine away from the fistulous tract. Of note, no hematuria was noted during stent placement. A Foley catheter was left in place and the patient was observed in the hospital for two nights wherein he remained afebrile with stable blood work.

\section{Outcome}

The patient's stent remained in situ for 4 weeks, at which time an ultrasonography of his left kidney and spleen
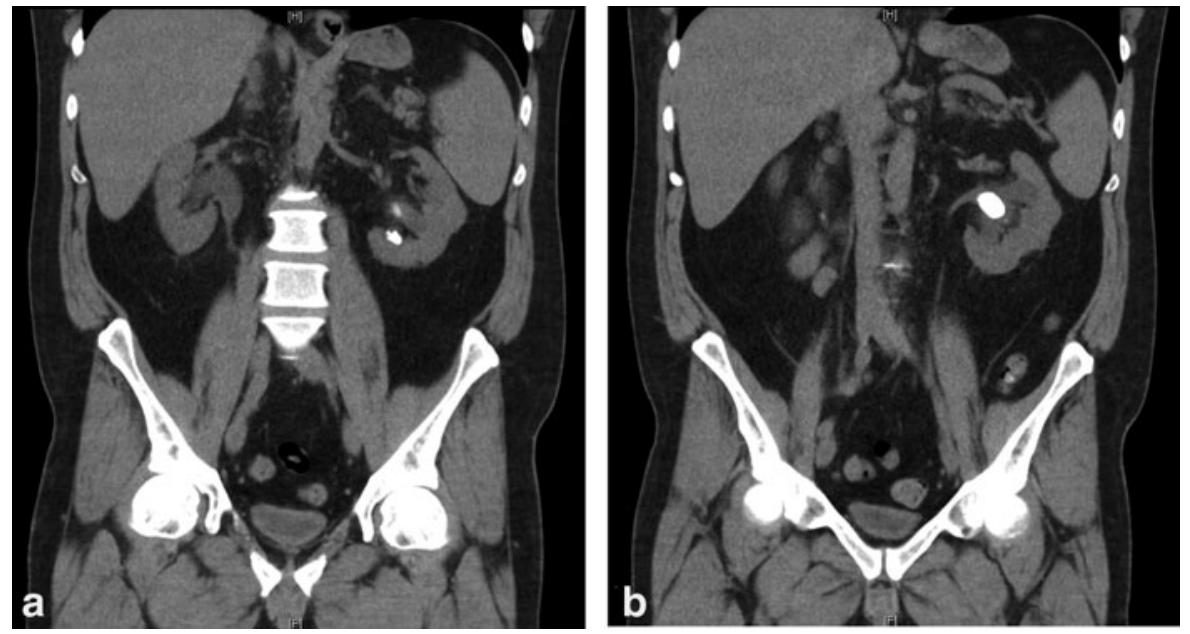

FIG. 1. Preoperative noncontrasted CT of abdomen/pelvis with coronal imaging of the $1.5 \mathrm{~cm}$ lower pole stone (a) and the $2.5 \mathrm{~cm}$ left renal pelvic stone (b). 

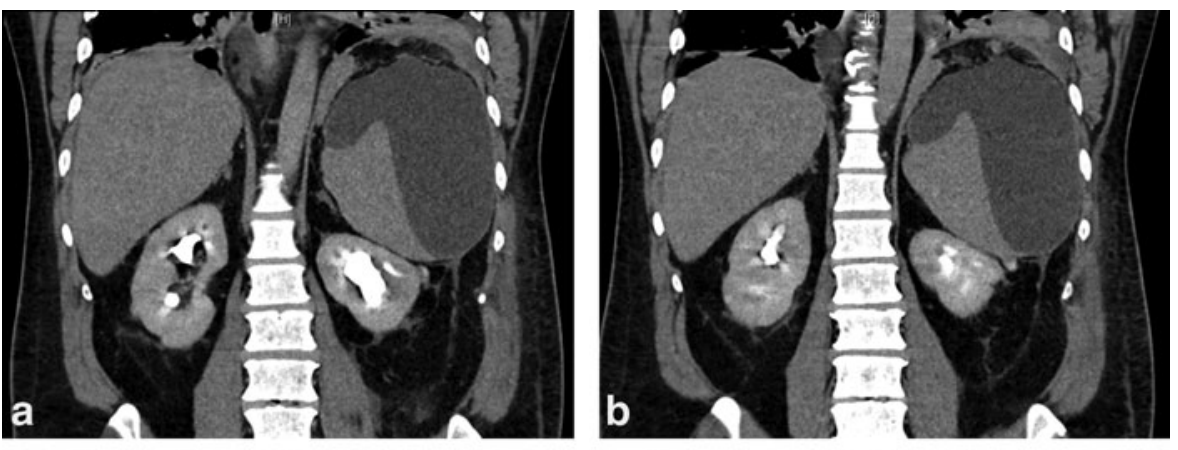

FIG. 2. Coronal images from CT urogram obtained in the emergency department showing fistulous connection from kidney to spleen with extravasation of contrast from anterior to posterior $(\mathbf{a}-\mathbf{d})$.
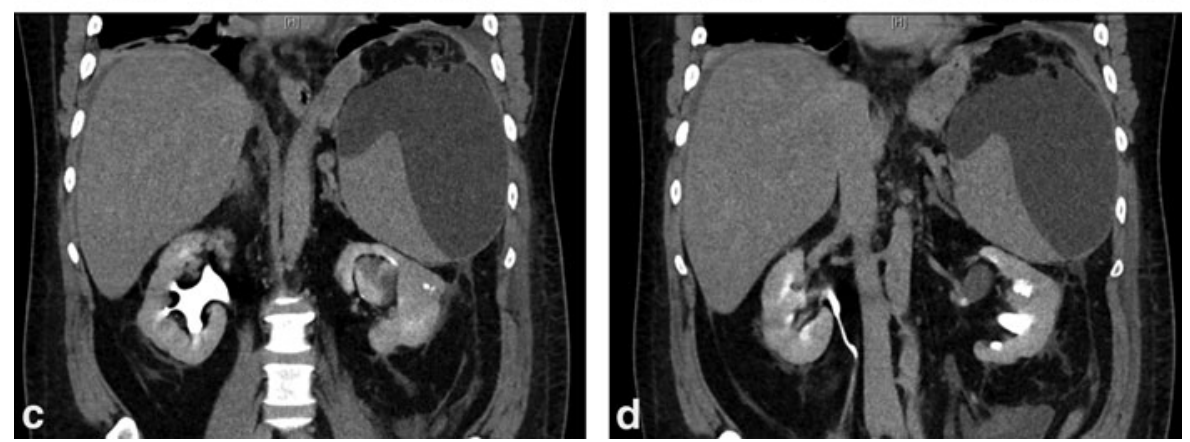

appeared anatomically normal, showing resolution of his splenic fluid collection. The stent was removed and follow-up CT scan 4 weeks later (6 weeks post-PNL) showed complete resolution of the urinoma and fistulous tract. (Fig. 3).

\section{Discussion and Literature Review}

Since the American Urology Association and the European Association of Urology guidelines recommend PNL as the first line treatment for large renal calculi, the utilization of this procedure has almost doubled in the United States, ${ }^{3}$ with this increase accompanied by a rise in complications rates. Fortunately, most PNL complications are minor and can be treated conservatively or with minimally invasive procedures. Major complications, although rare, could be devastating or even lethal.

One of the rare, but major, complications of PNL is splenic injury. So far, only 11 cases of splenic injury have been
FIG. 3. Noncontrasted $\mathrm{CT}$ of abdomen/pelvis with coronal $(\mathbf{a}, \mathbf{b})$ and axial (c, d) images showing no recurrence of the splenic urinoma 2 weeks after stent removal.
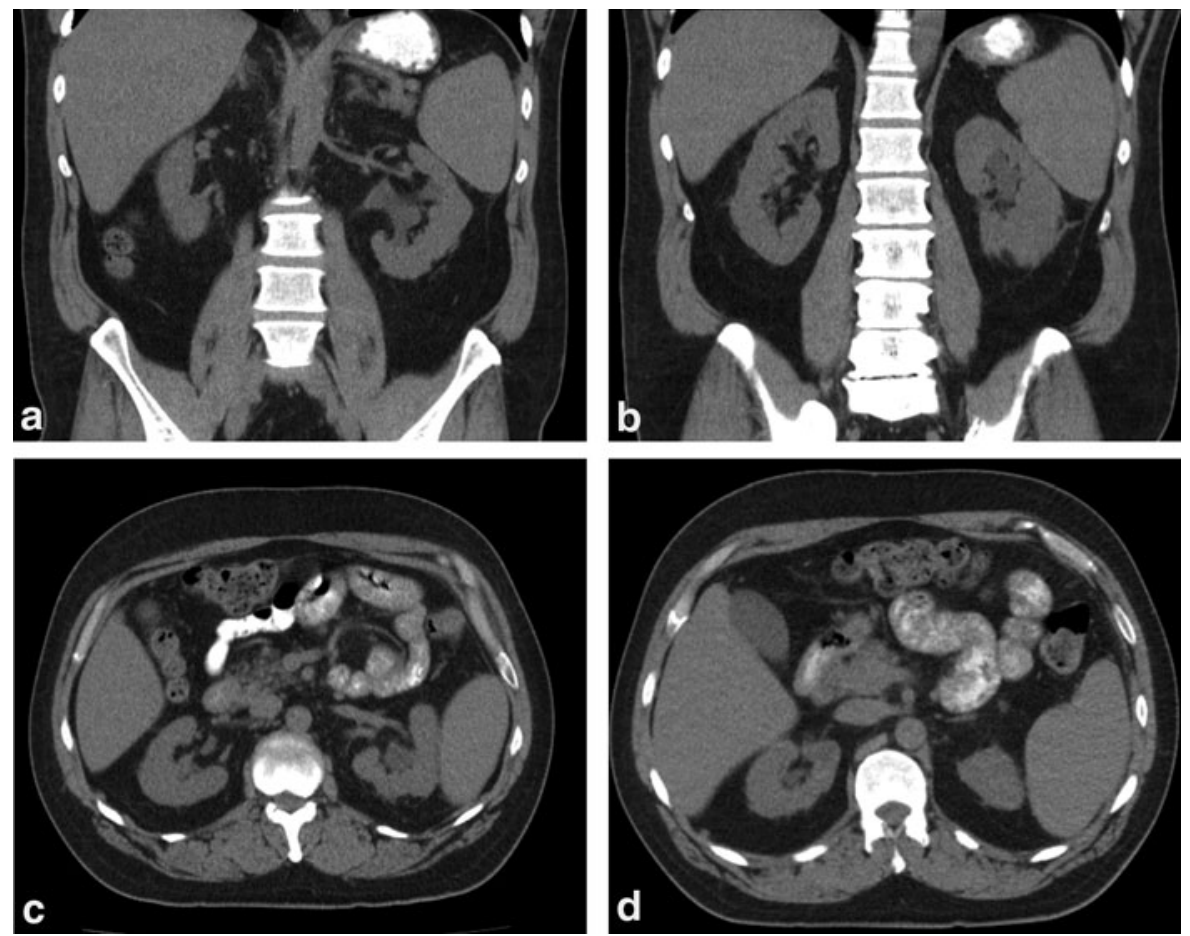
reported in the literature. ${ }^{4}$ Most of these injuries are hemorrhagic in nature and related to upper pole, especially supracostal, renal accesses during PNL. Although urgent exploratory laparotomy and splenectomy were needed in patients with hemodynamic instability, most cases of splenic injury were managed conservatively with transfusions, use of coagulant agents, and delayed nephrostomy tube removal. We herein present a new, nonhemorrhagic complication that involved the splenic capsule alone.

Our patient presented with delayed subcapsular splenic urinoma and a fistula between the urinoma and the renal collecting system. Injury to the splenic capsule most likely occurred during access needle placement, although another conceivable explanation is stretching/shearing of the splenic capsule during nephrostomy tract dilatation. It is notable that no significant bleeding was encountered during the PNL procedure. It is also possible an unrecognized injury to the splenic capsule was tamponaded by the access sheath and formalized into a fistulous tract after ureteral stent removal.

Extravasation from the renal collecting system after tubeless PNL is not uncommon. This complication is usually minimized by ureteral stent placement after stone removal. Although an internal stent was left in place for a week, it is possible that the patient continued to have urinary extravasation through a low-resistance path to the urinoma.

Choosing the optimal imaging study when the patient returned with flank pain was crucial to make the appropriate diagnosis. A noncontrasted CT would have shown a collection that could have been easily confused with hematoma, abscess, or perinephric urinoma. In this case, the patient may have received a percutaneous drainage tube that could have worsened his complication or resulted in splenic loss. This case highlights the importance of tailoring imaging studies to individual patient situations.

Equally important was the judicious decision to not drain this collection. Traditionally, patients with symptomatic urinomas after PNL are treated with percutaneous drainage. We elected not to place a percutaneous drain to avoid splenic injury and instead opted to facilitate internal drainage through placement of an internal ureteral stent. In addition, percutaneous drain placement was still an option if internal drainage alone could not resolve the urinoma. Although this precluded biochemical confirmation of urine in the collection, the reading radiologists felt confident that the collection was more consistent with urinary fistula than with hematoma.

When faced with uncommon complications, it is essential to keep patients fully informed of their status. Our patient was counseled extensively about the rarity of this complication and the potential need for further interventions. Fortunately, the patient did well after stent placement and did not need further management.

\section{Conclusion}

It is through reporting of unusual complications and their management that we advance our patients' urologic care. To our knowledge, this is the first report of a subcapsular splenic urinoma associated with splenorenal fistula as a complication of PNL. Prompt recognition and drainage with internal ureteral stent were sufficient to correct this unusual complication.

\section{Acknowledgment}

Institutional funding was used for the completion of this study.

\section{Disclosure Statement}

M.P. serves as a consultant for Boston Scientific. For all other authors, no competing financial interests exist.

\section{References}

1. Michel MS, Trojan L, Rassweiler JJ. Complications in percutaneous nephrolithotomy. Eur Urol 2007;51:899-906; discussion 906.

2. Seitz C, Desai M, Hacker A, et al. Incidence, prevention, and management of complications following percutaneous nephrolitholapaxy. Eur Urol 2012;61:146-158.

3. Mirheydar HS, Palazzi KL, Derweesh IH, Chang DC, Sur RL. Percutaneous nephrolithotomy use is increasing in the United States: An analysis of trends and complications. J Endourol 2013;27:979-983.

4. Ozturk H. Gastrointestinal system complications in percutaneous nephrolithotomy: A systematic review. J Endourol 2014;28:1256-1267.

\section{Address correspondence to: Eugene B. Cone, MD \\ Division of Urology \\ Duke Department of Surgery Duke University Medical Center DUMC Box 3707 Durham, NC 27710}

E-mail: eugene.cone@duke.edu

$\begin{aligned} & \text { Abbreviations Used } \\ \mathrm{CT} & =\text { computed tomography } \\ \mathrm{ED} & =\text { emergency department } \\ \mathrm{H} / \mathrm{H} & =\text { hemoglobin/hematocrit } \\ \mathrm{HU} & =\text { Hounsfield units } \\ \mathrm{PNL} & =\text { percutaneous nephrolithotomy }\end{aligned}$

Cite this article as: Cone EB, Jibara G, Wollin D, Preminger GM (2017) Subcapsular splenic urinoma and splenorenal fistula: a new complication of percutaneous nephrolithotomy, Journal of Endourology Case Reports 3:1, 134-137, DOI: 10.1089/cren.2017.0085. 